\title{
Benchmark Analysis for Robustness of Multi- Scale Urban Road Networks Under Global Disruptions
}

\author{
Wen-Long Shang, Member, IEEE, Ziyou Gao, Nicolò Daina, Haoran Zhang, Yin Long, Zhiling Guo* \\ and Washington Y. Ochieng
}

\begin{abstract}
To date immunity to disruptions of multi-scale urban road networks (URNs) has not been effectively quantified. This study uses robustness as a meaningful - if partial representation of immunity. We propose a novel Relative Area Index (RAI) based on traffic assignment theory to quantitatively measure the robustness of URNs under global capacity degradation due to three different types of disruptions, which takes into account many realistic characteristics. We also compare the RAI with weighted betweenness centrality, a traditional topological metric of robustness. We employ six realistic URNs as case studies for this comparison. Our analysis shows that RAI is a more effective measure of the robustness of URNs when multiscale URNs suffer from global disruptions. This improved effectiveness is achieved because of RAI's ability to capture the effects of realistic network characteristics such as network topology, flow patterns, link capacity, and travel demand. Also, the results highlight the importance of central management when URNs suffer from disruptions. Our novel method may provide a benchmark tool for comparing robustness of multi-scale URNs, which facilitates the understanding and improvement of network robustness for the planning and management of URNs.
\end{abstract}

Index Terms-Benchmark analysis, Global disruptions, Immunity, Robustness, Urban road networks.

\section{INTRODUCTION}

$\mathrm{H}$ ealth examination (HE) of urban transportation is a relatively new concept along with the establishment of urban physical examination, which is first proposed in Beijing urban master plan (2016-2035) [1]. As an important aspect of $\mathrm{HE}$, the ability of URNs to maintain normal operation when suffering from disruptions, that is, immunity of urban transportation to disruptions is receiving more attention.

This research is supported in part by the Chinese Scholarship Council (NO 201308060082), in part by Postdoctoral Research Foundation in China (NO. 2021M690341), in party by National Natural Science Foundation of China (NO. 72091513), in part by Natural Science Foundation of Beijing Municipality (NO. L211027) and in part by International Research Cooperation Seed Fund of Beijing University of Technology (NO. 2021A04).

W.L. Shang is with School of Traffic and Transportation, Beijing Jiaotong University, Beijing, China, Beijing Key Laboratory of Traffic Engineering, College of Metropolitan Transportation, Beijing University of Technology, Beijing, China, and Centre for Transport Studies, Imperial College London, London, United Kingdom (e-mail: shangwl_imperial@bjut.edu.cn).

Ziyou Gao is with School of Traffic and Transportation, Beijing Jiaotong University, Beijing, China (e-mail: zygao@bjtu.edu.cn).
Urban road networks (URN) are crucial infrastructure components of urban transportation and constitute an essential backbone underpinning most social and economic activities $[2,3]$. Road traffic networks and infrastructure are vulnerable to disasters [4-6], however, some of which, like extreme weather events or earthquakes, can be so disruptive as to lead to a complete system failure [7, 8]. In such circumstances the direct loss of life and economic loss arising from the disaster event itself are supplemented by indirect losses resulting from delays caused by the inability of emergency services and humanitarian agencies to use the road network effectively $[4,9]$.

Against this background, it is important to assess the level of immunity of URNs to major or global disruptions. Robustness of URNs is the traditional concept that the transportation planning community adopts to indicate the level of immunity. However, to the best of our knowledge, quantitative indices that assess and compare the ability of URNs to withstand global disruptions are deficient because they fail to account for all the attributes (road capacity, drivers' behavior and flow patterns) that enable a realistic representation of URNs and do not allow the comparison between multi-scale URNs under global disruptions. In this study, multi-scale URNs refer to the road networks with significant differences in the number of nodes and links.

The differences in types of network, the infrastructure they use and in the constraints and objectives applied to those networks have meant that it has been difficult for scholars to agree on a definition of what "robustness" actually means in the context of networked systems. In the context of electrical networks, robustness has been defined, variously, as "the degree to which a system or component can function correctly in the presence of invalid inputs or stressful environmental

Nicolò Daina is with School of Government and Public Policy, the University of Strathclyde, Glasgow, United Kingdom (email: nicolo.daina@strath.ac.uk)

Haoran Zhang and Zhiling Guo are with Centre for Spatial Information Science, The University of Tokyo, Japan (email: zhang ronan(a)csis.utokyo.ac.jp, guozhilingcc@esis.u-tokyo.ac.jp)

Yin Long is with Graduate School of Engineering, University of Tokyo, Japan (email: longyinutokyo@gmail.com)

Washington Y. Ochieng is with Centre for Transport Studies, Imperial College London, London, United Kingdom (email: w.ochieng@imperial.ac.uk).

* Author to whom correspondence should be addressed.

Manuscript received August 28, 2021 
conditions" [10] and "the ability to keep the network structure (function) intact when exposed to perturbations" [7]. In [11], Immers et al. consider robustness to be "the degree to which a system is capable of functioning according to its design specifications in the case of serious disruptions". In [12], Boccaletti et al. define it a network's ability to continue to operate when a proportion of its constituent elements are damaged. In [13], Schillo et al., meanwhile, define robustness as the ability to maintain "safety responsibilities", although they also see this as being related in a broad sense to system performance. In the context of URNs, robustness is defined in this study as the ability of a system to maintain its original performance when experiencing disruptions. It is also noteworthy that there are similarities between robustness and resilience. They both do not have unified definitions due to the diversity of network systems, and are both related to the ability of a system withstanding internal/external disturbances. However, they have significant differences. Robustness focuses on the ability of a system to maintain its original performance, which is inherent and static, while resilience emphasizes on the ability to 'bounce back' to the normal state of the system [14, $15]$.

There are many measures of robustness related to road networks, and the number of studies related to robustness of road networks is considerable relatively, which mainly focuses on either topology-based or traffic-based models. So far, however, no quantitative assessments of the robustness of URNs with different sizes have been able to capture the realistic features of networks experiencing global disruptions. Based on this, a relative area index (RAI) is proposed to conduct such benchmark analysis of multi-scale URNs, in order to examine the ability, namely, immunity of URNs against global disruptions.

The contribution of this paper is to propose a benchmark index, the relative area index (RAI), that can be used to measure the robustness of multi-scale URNs against different types of global capacity degradation. The RAI quantitatively captures the networks' ability to withstand internal or external global disruptions, and to keep performing at a satisfactory level. Based on this, the benchmark analysis of the robustness is conducted for six selected realistic urban road networks. Three types of weight parameters are utilized to model the nature of different global disruptions, and, in common with other network topological assessments of robustness, Spearman's ranking correlation test is applied to reveal potential correlations between proposed indices and weighted betweenness centrality. The aim of this study provides an effective tool for benchmark management of robustness planning on urban infrastructure against global disruptions, so as to facilitate the understanding to health state and immunity of URNs.

The paper is structured such that section 2 reviews previous work on the measurement of robustness, encompassing both the topological and operational sides of that question, and classifications of disruptions. Following this, section 3 explains the concepts and methodological approaches applied in this research, including weighted betweenness centrality and the relative area index based on User Equilibrium (UE) and System Optimum (SO) principles. Section 4 then presents a case study analysis to benchmark six urban road networks. Section 5 concludes the study, and discusses future works.

\section{LITERATURE REVIEW}

Until recently there was relatively little research into the robustness of transport networks to disruptions [16]. Nonetheless, the number of studies related to robustness of transport networks is now considerable. These mainly explore robust characteristics of networks based on either the topology of networks or traffic-based models. We review the literature on robustness in transport networks within these two classes. In addition, we also review the disruptions which may cause the negative impacts on URNs, so as to understand how they affect URNs.

\section{A. Robustness based on topology}

Topological indices of the robustness of networked systems focus on the network topology of networks without taking into account the distribution of any quantity transported by the network. Topological indices are based on complex network theory and include measures such as degree centrality, clustering coefficient, betweenness centrality, and the size of the largest connected component [17]. In essence these measures aim to reflect how efficiently the underlying network functions [18-22], and in that sense they are intrinsically also measures of robustness. In [23, 24], Shang et al. and Callaway et al. explain these indices more fully.

In [22], Albert et al. consider measures such as the change of diameter, the size of the largest cluster and the average size of isolated clusters as means of assessing the tolerance of networks to disruptions, whether deliberate or random. In [18], Holme et al. find that removing nodes from complex networks in a descending order of recalculated degree and betweenness has a more adverse effect on robustness than when nodes are removed in a descending order of initial degree and betweenness. Similarly, in [19], Crucitti et al. focus on shortest paths as a measure of the efficiency of simulated networks, in [25], Crucitti et al. examine the consequences for the global efficiency of electric networks when nodes are either removed randomly or in descending order from the largest load. In the context of road networks, meanwhile, in [26], Sakakibara et al. propose a topological index based on network dispersiveness/concentration to evaluate robustness in disaster situations, aiming to minimize the isolation of districts in such circumstances. In [27], Sun et al., meanwhile, focus on betweenness: i.e. the measure of how often a given node is on the shortest route between any pair of nodes. They show how the robustness of an air traffic control network can be measured using a statistical distribution of the betweenness of all the nodes in that network. Specifically, they argue that networks with a higher number of nodes with small betweenness will be more robust against individual failures than a network with fewer such nodes.

To identify the critical factors, in [28], Wang et al. utilize the 
area of radar diagrams for ten topological metrics to assess overall robustness of 33 realistic metro networks, as a result, transfer stations and long link sections are identified as two important factors of robustness of metro networks. In [29], Clark et al. have developed an approach to assess robustness and resilience of National Airspace System (NAS), the relative size of largest connected cluster in the NAS is used to quantify the tolerance to loss of critical functions, and they demonstrate that centrality measures are a good platform for supporting restoration. In addition, in [30], Zhang et al. explore the robustness and vulnerability of Shanghai metro network by calculating its network efficiency from topological perspective when removing nodes.

Although many studies into network robustness, such as those above, adopt topological approaches to the problem, topological indices are unable to capture realistic capacity, traveler behaviors and flow patterns in urban road networks (URNs).

\section{B. Robustness based on traffic-based models}

In the context of URNs, therefore, operational indices are also often used to measure robustness. These take into account travel demand, driver behaviors [31] and link capacity. In this strand of the studies, in [32], Scott et al. proposed a new Network Robust Index (NRI), encompassing network flows, link capacity and network topology. This seeks to locate the links that have the most impact on the efficiency of a network by estimating the overall impact on travel time as individual links are removed from the network. This study then compares the results from the NRI calculation to those of the traditional measure of critical links, the volume/capacity (V/C) ratio, revealing that NRI provides better planning solutions for congested networks than V/C. In [33], Sullivan et al. extend the earlier work by proposing the Network Trip Robustness (NTR) as a measure of the system-wide robustness of transportation networks in circumstances where links experience reductions in capacity. In [34], Nagurney and Qiang, meanwhile, propose a network efficiency measure by capturing demand, flows, costs and user behaviors from a traffic user equilibrium model, and then use the measure to access the robustness of transport networks and rank the impact of individual links on the efficiency of the whole network. In their subsequent work [35], Nagurney and Qiang then use the relative change in the proposed measure as a proxy for the robustness of the network to a gradual reduction in the capacity of all its links. Indeed, this study focuses on degrading link capacity rather than removing links, which distinguishes Nagurney and Qiang's work from that other previous studies. Thereafter, in [36], Nagurney and Qiang develop another measure of robustness to degraded network links, which they dub the "relative total cost index". That approach picks up the travel behaviors associated with User Optimization (UO) and System Optimization (SO) to derive the overall cost of a given journey. In [37], Zhao et al. utilize this relative total cost index based on stochastic user equilibrium to investigate the robustness and Braess paradox of networks.

Although there are not many measures of robustness based on
TABLE I

CATEGORIES OF ROAD DISRUPTIONS

\begin{tabular}{lll}
\hline \hline Frequent & Unusual \\
\hline Predicted & $\begin{array}{l}\text { Predictable changes in } \\
\text { supply and demand, } \\
\text { planned or regular } \\
\text { maintenance }\end{array}$ & $\begin{array}{l}\text { Major public events, bad } \\
\text { weather, strikes }\end{array}$ \\
Unpredicted & $\begin{array}{l}\text { Minor road traffic } \\
\text { accidents, facility } \\
\text { failures }\end{array}$ & $\begin{array}{l}\text { Natural disasters, deliberate } \\
\text { damage or hostile acts (e.g. } \\
\text { terrorist, military or cyber } \\
\text { attacks) }\end{array}$ \\
\hline \hline
\end{tabular}

operational indices, they are able to apprehend features such as travel demand, link capacity and driver behavior more realistically. In addition, the quantitative measures of robustness of road networks with different sizes against global capacity degradation are highly insufficient.

\section{Classification of disruptions}

There have been many studies into disruptions of URNs, such as transit strikes [38-40], bridge closure/collapse [41-43], special events [44, 45] and earthquakes [46-48]. Broadly, these disruptions can be categorized as expected or unexpected in nature $[49,50]$, and as occurring in a regular, predictable way, or not [11]. TABLE I that categorizes potential disruptions is shown below.

While all these disruptions have the potential to result in the poor functioning of URNs, and some may even result in a risk of system collapse[51], this study focuses on those that have the potential for more serious effects on the road infrastructure, i.e. those that could negatively affect an URN at global level. These disruptions include extreme weather conditions and other natural calamities [52]. Furthermore, these disruptions affect transport networks in different ways: some, such as torrential rain, lead to reduce capacity, while others, such as an earthquake, may remove nodes/links[53].

Based on above review, this study mainly concentrates on the disruptions which impact the URNs by means that may cause a global capacity degradation. The study specifically seeks to examine the inherent robustness of URNs against global disruptions caused by extreme weather condition or natural calamities that have the potential to cause high levels of destruction to the road infrastructure.

\section{Methodology And DataBASE}

The methodology applied in this study is (1) to use the proposed relative area index (RAI), derived from user equilibrium (UE) and system optimum (SO), to quantify the robustness of URNs; and (2) to use weighted betweenness centrality $(\mathrm{WBC})$ as a baseline.

In order to show the calculation process of RAI in full, the UE and SO is quickly recapped, although the discussions of them are standard. More detailed information is referred to [54] and [55]. 


\section{A. The relative area index under distinct principles}

Assuming in a directed network $G(N, A), N$ is a set of nodes, $A$ is a set of links, $W$ is a set of origin-destination (OD) pairs, $(i, j) \in W$ indicates a specific OD pair and $T_{i j}$ is fixed travel demand. In addition, the paths for OD pair $(i, j) \in W$ is the set $P_{i j}$, the unit travel cost to complete path $p \in P_{i j}$ is $c_{p}$, and the flow on path $p$ is represented as $h_{p}$.

The link flows, $f_{a}$, are associated with the path flows and are represented in the equation:

$$
f_{a}=\sum_{p \in P} \delta_{a p} h_{p} \quad \forall a \in A
$$

where $\delta_{a p}$ is a link-path incident matrix:

$$
\delta_{a p}=\left\{\begin{array}{lr}
1 & \text { if } a \text { belongs to } p \\
0 & \text { if } a \text { does not belong to } p
\end{array}\right.
$$

The cost of travelling on a given path in the network is articulated in (2):

$$
c_{p}=\sum_{a \in A} \delta_{a p} c_{a}(f) \quad \forall p \in P
$$

In the above, $c_{a}$ is a unit cost function that is intrinsically dependent on the flow along the link-represented as $f=$ $\left(f_{a}: a \in A\right)$. This is then combined with the well-known US Bureau of Public Roads [56] (BPR) link performance function to create the link function shown in (3):

$$
t\left(f_{a}\right)=t_{a}\left(1+\alpha\left(\frac{f_{a}}{K_{a}}\right)^{\beta}\right) \forall a \epsilon A
$$

where $f_{a}$ denotes flows on each link $a \in A, t_{a}$ and $K_{a}$ are, respectively, the free flow travel time and capacity of link $a$; and $\alpha, \beta$ are positive constants.

The following flow conservation must hold:

$$
T_{i j}-\sum_{p \in P_{i j}} h_{p}=0 \quad \forall(i, j) \in W
$$

Then the set of feasible flows is shown below:

$$
\gamma=\left\{h \geq 0: T_{i j}-\sum_{p \in P_{i j}} h_{p}=0 \forall(i, j) \in W\right\}
$$

As (5) describes, the sum of the flows across all the individual paths of the O-D pair $(o, d)$ equates to the overall travel demand for the $(o, d)$.

\section{1) Total cost under User Equilibrium (UE)}

The Wardrop [57] proposed a principle that a certain equilibrium is reached when individual drivers selfishly pursue the maximization of their personal interests. This concept, often referred to as "User Equilibrium" (UE) can be expressed mathematically using the optimization below [58].

$$
\operatorname{Min} Z=\sum_{a} \int_{0}^{f_{a}} c_{a}\left(f_{a}\right) d f
$$

Subject to

$$
\begin{aligned}
& \sum_{p \in P_{i j}} h_{p}=T_{i j} \quad \forall(i, j) \in W \\
& f_{a}=\sum_{i} \sum_{j} \sum_{p} \delta_{a p}^{i j} h_{p}^{i j} \quad \forall a \in A
\end{aligned}
$$

$$
\begin{array}{cr}
h_{p}^{i j} \geq 0 & \forall p \in P_{i j}, \forall(i, j) \in W \\
f_{a} \geq 0 & \forall a \in A
\end{array}
$$

The total cost $\left(T C_{U E}\right)$ of travel in a network that is at its user equilibrium is given by:

$$
T C_{U E}=\sum_{a \in A} f_{a}^{*} c_{a}\left(f_{a}^{*}\right)
$$

The equations above include the objective (6), and a series of constraints, respectively, for conservation (7) and (8), and nonnegative flows (9). The link cost function increases monotonically such that the link flow pattern of UE is unique [59] and the objective of the program is convex. A fixed point algorithm (FPA) is applied to the calculation of UE solutions $f_{a}^{*}$. For the detailed procedure for this refer to [55].

\section{2) Total cost under System Optimum (SO)}

Another significant contribution by Wardrop [57] lies in his second principle of traffic assignment: System Optimum (SO). This is the process whereby centralized controllers assign drivers to routes in such a way as to minimize the total travel costs incurred by all drivers [60]. The System Optimum can be expressed mathematically using the nonlinear optimization below:

$$
\operatorname{Min} Z=\sum_{a} f_{a} c_{a}\left(f_{a}\right)
$$

Subject to

$$
\sum_{p \in P_{i j}} h_{p}=T_{i j} \quad \forall(i, j) \in W
$$




$$
\begin{aligned}
& f_{a}=\sum_{i} \sum_{j} \sum_{p} \delta_{a p}^{i j} h_{p}^{i j} \quad \forall a \in A \\
& h_{p}^{i j} \geq 0 \quad \forall p \in P_{i j}, \forall(i, j) \in W \\
& f_{a} \geq 0 \\
& \forall a \in A
\end{aligned}
$$

Here, the total cost $\left(T C_{S O}\right)$ of travel in a network that is at its system optimum is given by:

$$
T C_{S O}=\sum_{a \in A} f_{a}^{*} c_{a}\left(f_{a}^{*}\right)
$$

In this study, FPA is used to calculate the SO solutions $f_{a}^{*}$.
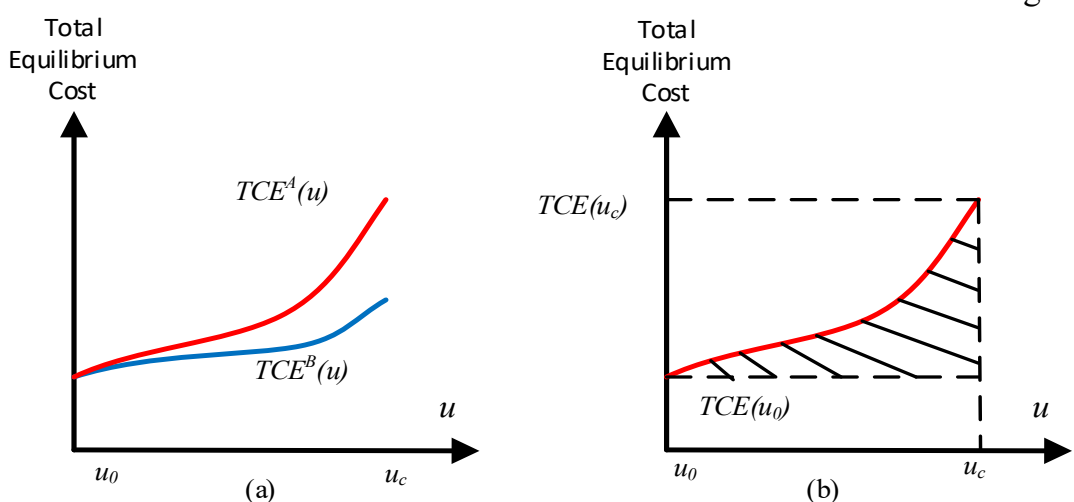

(b)
3) Robustness key performance indicator (KPI): Relative Area Index (RAI)

We use the above principles of UE and SO as the basis for our proposed Relative Area Index (RAI) to explore the robustness of multi-scale URNs.

Various levels of capacity degradation for global URNs are taken into account. Let $u$ be a capacity degradation parameter (CDP) between $u_{0}$ and $u_{c}$. When $u$ is equal to $u_{0}$, URNs do not suffer from any disruptions and the capacity of URNs stays maximum. $u_{c}$ denotes URNs that are subject to a capacity degradation level which may cause a significant increase of total travel cost. Furthermore, TCE $(u)$ is denoted as the curve of total cost based on equilibrium state of URNs suffering from capacity reduction of $u$, which can be calculated by (6)-(10) and (11)-(14) according to different equilibrium principles: UE and SO. Some examples that depict the evolution of network performance under different levels of global disruptions are shown in Fig. 1 below.

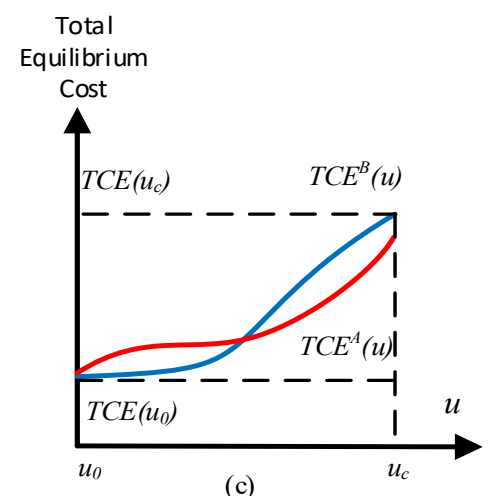

(c)

Fig. 1. (a): two examples of the function $\boldsymbol{T C E}(\boldsymbol{u})$ : the lower (blue) is a more robust network than the upper (red) one. (b): illustration of the relative area (the ratio of the shadowed area to the area within the box) as an indicator of by how much the network capacity is reduced. (c): illustration that some form of weighting is needed since two different curves can return the same relative area.

In order to capture and quantify deterioration of global capacity of URNs, we consider the area formed by the TCE $(u)$ curve. The two line segments (Fig. 1. (a)) represent different evolution curves of total cost for different URNs suffering from global disruptions. Two functions, $T C E^{A}(u)$ and $T C E^{B}(u)$ corresponding to URN $A$ and URN $B$, show that URN $B$ is more robust than URN $A$. The shadow area (Fig. 1. (b)) reflects how much the network-level cost deviates from the original performance (total cost without disruptions) as the network capacity is reduced. Therefore, the smaller the shadow area is, the more robust the URN is against global disruptions. Because two different $T C E(u)$ curves may yield the same area (Fig. 1. (c)), a weighting parameter $w(u)$ is introduced to distinguish such case. $w(u)$ can be assigned different values which depends on the types and nature of disruptions. For example, if the global disruptions are caused by daily maintenance, the capacity degradation is mild, and the smaller weights will be assigned to $w(u)$ with larger $u$. Therefore, as shown in graph (c) of Fig. 1, the city A (red line) has a larger weighted area than city B (blue line) and thus is less robust. In this study, three types of weighting parameters are used. The first type assigns the equal values to $w(u)$, which corresponds to the disruptions that may impact URNs to the same extent as network capacity deteriorates, such as regular maintenance activities. The second type assumes that the larger weights correspond to the smaller values of $u$, and this type of disruptions tend to cause the cascading failure, such as traffic accidents at the critical parts of URNs. The third type assumes that the larger values of $u$ use higher weights, which is related to the disruptions that have accumulative impacts on URNs, such as continuous rainfall or snowfall. These three types of weighting parameters can reflect the different ways that disruptions impact URNs, and will facilitate the exploration of robustness of URNs against global capacity degradation. Following this, Relative Area Index (RAI) for URNs is formulated as follows:

$$
R A I=\frac{\int_{0}^{u} w(u)[T C E(u)-T C E(0)] d u}{\int_{0}^{u} w(u) T C E(0) d u}
$$

The numerator of (15) is the weighted area with weights, which measures how much the curves deviate from the original performance of URNs at equilibrium state. The equation's denominator, however, serves to normalize the area. This makes it possible to examine by how much the performance of 
a road network has reduced compared to its undisturbed performance. For a given URN, the larger the RAI is, the greater the increase in the cost associated with the URN when the network capacity drops, thus the URN is less robust for global disruptions. In the study, we utilize the total travel cost and relative area to measure the performance and robustness of URNs, respectively, and (15) is able to illustrate the relationship between network performance and the robustness of URNs well.

Since the RAI used in this study is based on UE and SO principles, the proposed index can be divided into RAI-UE and RAI-SO respectively.

This RAI index is very similar to an index proposed by [23]. The latter index is more focused on the capacity reduction at the nodal level and is based on the maximal flow model of European air traffic network, whereas this novel RAI is based on the UE and SO traffic model of URNs and more concentrates on the robustness of URNs suffering from global disruptions at network level.

\section{B. Weighted betweenness centrality}

Betweenness centrality $(B C)$ is another important topological concept for exploring the robustness of urban road networks [61]. $B C_{i}$ is defined at each node $i$, as:

$$
B C_{i}=\sum_{j, k \in V} \frac{N_{j k}(i)}{N_{j k}}
$$

where $N_{j k}(i)$ is the number of shortest paths passing through the node $i$, and $N_{j k}$ is the total number of shortest paths between any pair of nodes.

The edge weight is the actual distance of the link so it is called weighted Betweenness centrality. Depending on the needs of each particular piece of research, however, the edge weight of betweenness centrality may use different types of values, such as flow on each link and physical distance. Since there is usually a lack of data on link flows, physical distance is generally used as the weight of links [62], and we follow this approach here.

Betweenness measures how often a given node connects a network's most critical paths; thus $B C_{i}$ is the importance of that given node in those networks [63]. This helps to identify the key components and the robustness of networks subject to disruptions in specific localities.

\section{Database}

The novel RAI introduced above is used to assess the robustness of multi-scale URNs suffering from global capacity degradation. Data related to URNs are mainly obtained from a website ${ }^{1}$ frequently used for transportation problems. Since the available data sources are very limited, and since it is computationally expensive to calculate $\mathrm{UE}$ and $\mathrm{SO}$ solutions for large URNs, we selected networks from Anaheim, Friedrichshian centre, Prenzlauerberg centre, Tiergarten centre,
Mitte centre, and Mitte-Prenzlauerberg-Friedrichshain (MPF) centre for the benchmark analysis of the robustness. These URNs are appropriate for exhibiting how RAI effectively examines the robustness of multi-scale URNs. In this study, we mainly focus on single-mode (car) traffic, and the OD demand is assumed to be fixed. In reality, OD demand is probably uncertain and varies over time, but on the one hand, real-time OD demand data is not available from the dataset, on the other hand, the number of traffics in cities between origins and destinations tends to be stable for a relatively long term. Therefore, fixed OD demand is appropriate to be used to assess robustness here.

\section{CASE STUDY}

This section applies the methodology described above to determine the robustness performance of the six selected urban road networks in the context of global capacity degradation. In the study, the size of the selected URNs is appropriate for frequently calculating total travel cost at equilibrium, and global disruptions can be defined as events that cause the capacity degradation of URNs at global level, such as extreme weather conditions. Although there are many ways to assess the robustness of a network, as explained in section 2, these are unable to quantitatively examine and compare the robustness performance of multi-scale urban road networks experiencing disruptions, taking into account travel demand, link capacity, driver behaviors and so on. Thus the relative area index (RAI) has been developed here to conduct benchmark analysis of robustness for URNs with different sizes, and then make comparisons to the results of WBC.

\section{A. Benchmark analysis of robustness based on RAI}

As can be seen from Fig. 2, it presents the relative TUE and TSO curves for the six selected networks when they are subject to global capacity deterioration. Here TUE and TSO refer to the total travel cost at $\mathrm{UE}$ and $\mathrm{SO}$ equilibrium under different levels of capacity degradation, respectively. The values of TUE and TSO are normalized by their values without any capacity reductions.

In this section, three sets of RAI are calculated using three different weighting parameters (WP), which emphasize the impact of the different types of disruptions on the network performance. The first WP takes the equal values, and is denoted as ' $W P I$ ' in Fig. 3. As for the second and third WP, lower (higher) weight values are assigned to higher capacity degradation and higher (lower) values are assigned to lower capacity degradation, and are denoted as WP2 and WP3, respectively. The values of $W P 2$ and $W P 3$ vary from 12 to 0 and 0 to 12 accordingly so as to distinguish the impact of different levels of capacity deterioration. In fact, range of values depends on the range of capacity reduction. Assigning values to these weights is an interesting research topic, but we are not going to discuss it due to the limitation of space and the scope of this study. In this study, we just simply use the percentage of network capacity reduction to indicate the impacts of global

\footnotetext{
${ }^{1}$ http://www.bgu.ac.il/ bargera/tntp/
} 
disruptions on the URNs, which are all based on previous experience rather than precise calibration. As the advance of Information and Communication Technology (ICT), advanced

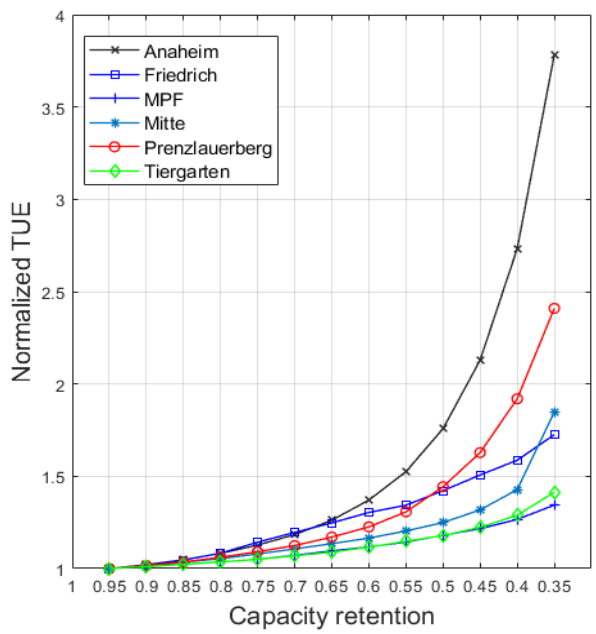

sensors have potential to accurately estimate the global capacity degradation of URNs, which would be our focus in the future study.

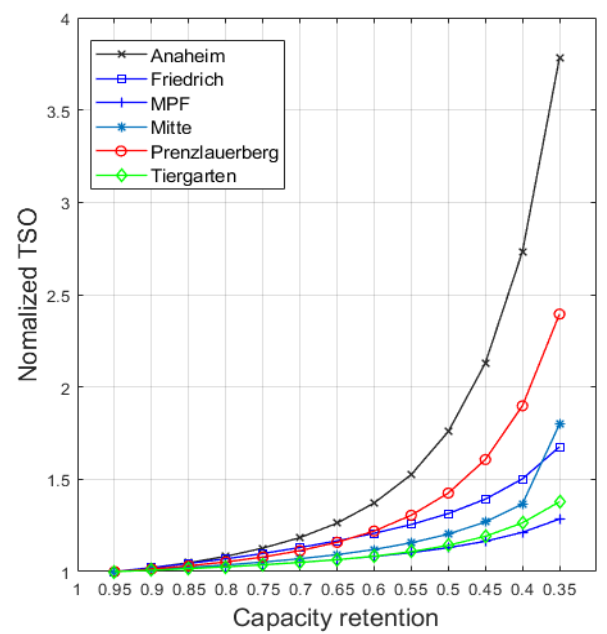

Fig. 2. Normalized TUE and TSO curves for six urban road networks subjected to a global reduction in capacity

The figure shows global capacity reduction ranges from $0 \%$ to $65 \%$ (i.e. a $100 \%-35 \%$ capacity retention rate). The reason why the capacity reduction is only within the range $0 \%$ to $65 \%$ is that this range is appropriate for frequent calculation of UE and SO equilibrium, and examples here are just used for presenting how this novel RAI works. This figure shows the curves of normalized total cost of UE and SO for selected URNs at different levels of global capacity degradation. It is evident from the normalized TUE and TSO data in Fig. 2 that the network whose total cost varies the most when it experiences capacity reductions is that of Anaheim. On the other hand, the MPF network is relatively close to original total cost, and curves of other networks such as Friedrichshian and
Prenzlauberg, cross each other. If the WPs at each level of capacity reductions are equal (WP1 case), we may say the robustness of Anaheim is the worst and WPF is the most robust among the six selected URNs suffering from global capacity degradation. However, it is difficult to tell which of the other URNs has better robustness since some of the curves overlap or cross. If we turn then to the WP2 and WP 3 cases, it becomes still more difficult to distinguish the robustness. In these cases, the RAIs are calculated based on (16) so as to quantify the magnitude of deviations as accurately as possible, by taking into account the nature of different types of disruptions. All RAI values for the different types of WP are shown in Fig. 3.

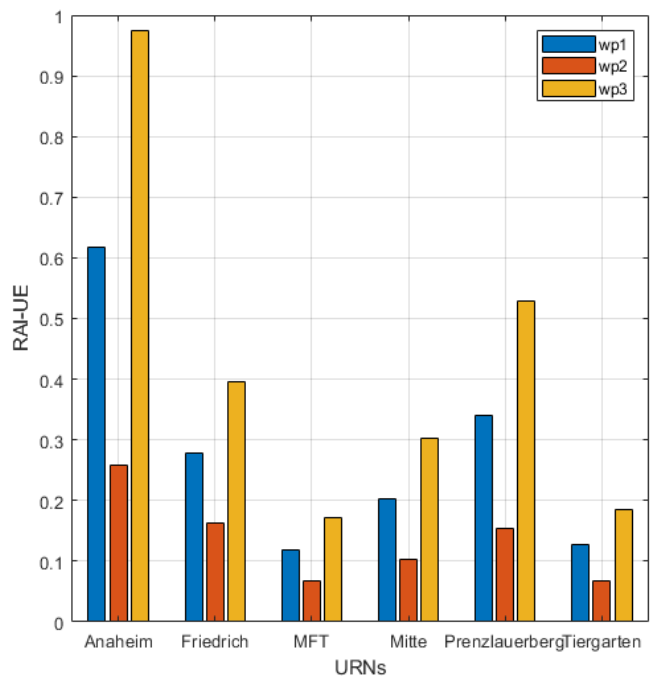

Fig. 3. RAIs of URNs based on TUE and TSO with three types of WP

As can be seen from Fig. 3, three sets of RAI for selected URNs are presented based on UE and SO principles, and the ranking based on RAI-SO is approximately consistent with that

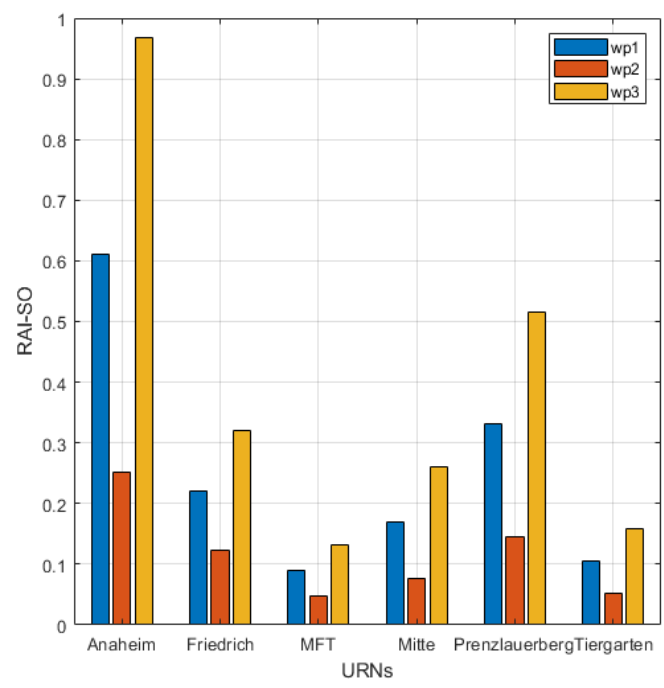

based on RAI-UE. In the principle of UE, RAIs for three types of WP show that Anaheim has the worst robustness and MFT is assessed as the most robust against the global capacity 
degradation from $0 \%$ to $65 \%$. Overall, rankings of RAI in all three cases of WP are nearly identical, and in the cases of WP1 and WP3, the following robust URN is Tiergarten, Mitte, Friedrichshian and Prenzlauerberg rank third, fourth and fifth robust. In the case of $W P 2$, due to the fact that lower capacity degradation takes higher weight, the ranking of robustness based on RAI is slightly different from the cases of WP1 and $W P 3$, and Friedrichshian is measured as the second worst robust, followed by Prienzlauerberg, Mitte and Tiergarte.

In contrast to UE, the rankings of RAIs based on SO for all three types of WP are completely identical, MFT network shows the best robustness among all selected URNs, the following are Tiergarten, Mitte, Friedich and Prienzlauerberg, while Anaheim seems to have worst robustness against global disruptions. Due to the fact that RAI is calculated by considering many realistic characteristics, it is very difficult to distinguish which factors may impact the robustness of Anaheim most greatly, but network topology plays an important role in its robustness. The rankings between RAI-SO and RAIUE are approximately the same except the fourth and fifth places of RAI-UE with WP2. In addition, all values of RAIs based on UE and SO with three types of WP are summarized in TABLE II, and it demonstrates that differences of robustness are not completely related to the size of URNs (number of nodes and links), that is, the immunity of URNs to global disruptions is not necessarily correlated with the scale of URNs. Intuition would suggest that the larger the scale of a URN the more robust it is likely to be, since larger URNs are likely to have more spare capacity [64]. Our finding goes against this intuition, however. The reason for this non-intuitive result depend on the complex nature of urban traffic and reflects realistic factors such as flow patterns, network topology and user behaviours.

Overall, as can be seen in TABLE III, different types of WPs appear to have little impacts on the ranking of robustness. Also, the rankings of RAIs under principles of UE and SO are nearly identical. However, it is worth noting that RAIs under the principle of SO are smaller than those under UE (as shown in Fig. 4), which implies that when the URNs suffer from global disruptions, global management from central entities may achieve better robustness than all individual users selfishly pursuing personal benefits maximum. In fact, the differences of RAI under UE and SO principles are very similar with Price of Anarchy [65], which is also used to measure the inefficiency of the systems caused by selfish behaviors.

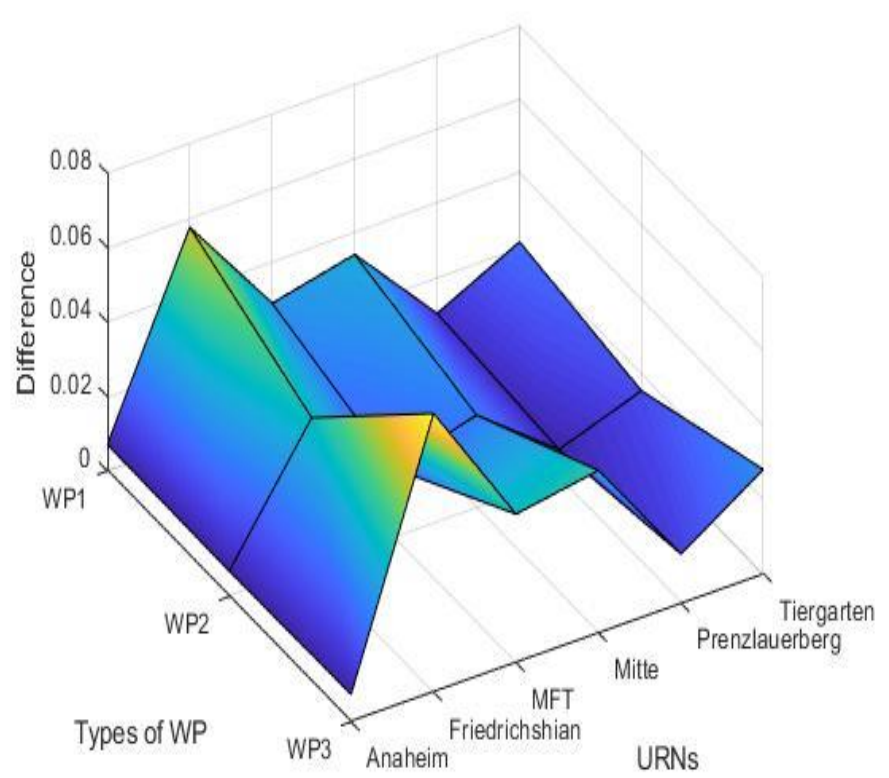

Fig. 4. Differences of RAIs between UE and SO

\section{B. Comparisons between RAI and WBC}

RAI-UE and RAI-SO are compared in TABLE II with the $W B C$, which, as explained in section 3 , has been used to measure the robustness of networks [27].

As can be seen, TABLE III shows how these networks rank in terms of their robustness.

TABLE II

Summary of RAIs and WBC for six urban road networks ( $N$ denotes the number of nodes and $L$ represents the number of links).

\begin{tabular}{|c|c|c|c|c|c|c|c|c|c|}
\hline \multirow{2}{*}{ Networks } & \multirow{2}{*}{$N$} & \multirow{2}{*}{$L$} & \multicolumn{3}{|c|}{ RAI-UE } & \multicolumn{3}{|c|}{ RAI-SO } & \multirow{2}{*}{$W B C$} \\
\hline & & & WP1 & WP2 & WP3 & WP1 & WP2 & WP3 & \\
\hline Anaheim & 416 & 914 & 0.6171 & 0.2590 & 0.9752 & 0.6100 & 0.2522 & 0.9678 & 0.0316 \\
\hline Friedrichshian & 224 & 523 & 0.2786 & 0.1625 & 0.3947 & 0.2211 & 0.1225 & 0.3198 & 0.0406 \\
\hline MPF & 975 & 2184 & 0.1196 & 0.0671 & 0.1721 & 0.0905 & 0.0487 & 0.1322 & 0.0124 \\
\hline Mitte & 398 & 871 & 0.2029 & 0.1021 & 0.3036 & 0.1686 & 0.0773 & 0.2600 & 0.0309 \\
\hline Prenzlauerberg & 352 & 749 & 0.3409 & 0.1538 & 0.5280 & 0.3305 & 0.1462 & 0.5147 & 0.0364 \\
\hline Tiergarten & 361 & 766 & 0.1266 & 0.0679 & 0.1854 & 0.1050 & 0.0525 & 0.1575 & 0.0352 \\
\hline
\end{tabular}

TABLE III

Robustness rankings of urban road networks

\begin{tabular}{|c|c|c|c|c|c|c|c|}
\hline \multirow{2}{*}{ Rank } & \multicolumn{3}{|c|}{ RAI-UE } & \multicolumn{3}{|c|}{ RAI-SO } & \multirow{2}{*}{$W B C$} \\
\hline & WP1 & WP2 & WP3 & WP1 & WP2 & WP3 & \\
\hline
\end{tabular}




\begin{tabular}{|c|c|c|c|c|c|c|c|}
\hline 1 & MPF & MPF & MPF & MPF & MPF & MPF & MPF \\
\hline 2 & Tiergarten & Tiergarten & Tiergarten & Tiergarten & Tiergarten & Tiergarten & Mitte \\
\hline 3 & Mitte & Mitte & Mitte & Mitte & Mitte & Mitte & Anaheim \\
\hline 4 & Friedrichshian & Prenzlauerberg & Friedrichshian & Friedrichshian & Friedrichshian & Friedrichshian & Tiergarten \\
\hline 5 & Prenzlauerberg & Friedrichshian & Prenzlauerberg & Prenzlauerberg & Prenzlauerberg & Prenzlauerberg & Prenzlauerberg \\
\hline 6 & Anaheim & Anaheim & Anaheim & Anaheim & Anaheim & Anaheim & Friedrichshian \\
\hline
\end{tabular}

The $W B C$ can measure how frequently a given node of URNs can be passed by the shortest paths of all OD pairs [3]. According to [27], a network is more robust if the proportion of nodes with high $B C$ is lower, which means the $W B C$ of a network can be related to the robustness of the network. TABLE III shows a significant difference between the raking obtained using RAI-UE or RAI-SO and those obtained WBC. However, RAI-UE, RAI-SO and $W B C$ all identify MPF the most robust URN. The Spearman correlation coefficients of rankings between them are not very high, and all below 0.55 . Here Spearman correlation coefficient is used to measure how well the monotonic relationship between two variables exists [66]. Although betweenness centrality can measure the "importance" of a given node in a network, it does so from the perspective of network topology alone, and is thus not a sophisticated measure encompassing the characteristics that realistically inform URNs, such as capacity, flow propagation and driver behaviors. This weakness is addressed by RAI-SO and RAI-UE. The RAI also better quantifies the robustness of urban road networks experiencing global capacity degradation. Altogether, therefore, the proposed RAI offers a more complete and realistic assessment of the robustness of urban road networks. The differences of robustness among these six URNs root in distinctive network structure and spatial layout, different distributions of travel demand on different OD pairs, and nonidentical travelers' behaviors. These heterogeneous characteristics jointly lead to different robustness of the URNs, and we may explore this in the future given that the current datasets are very limited.

Through such comparisons, the robustness rankings of these multi-scale URNs against global disruptions are presented and analyzed, which may shed light on the planning and management of urban traffics.

\section{CONCLUSION}

In the context of Health Examination (HE) of urban transportation, in order to measure the immunity of urban road networks (URNs) against disruptions which may cause the global capacity degradation, this study proposed a novel index, the relative area index (RAI), that quantitatively measures robustness and allows to conduct, for the first time, robustness benchmarking amongst URNs with different sizes. This research sheds light on how to quantify the immunity of urban transportation against global disruptions. The proposed RAI provides different insights from distinct perspective to assess the robustness of multi-scale URNs, and compared to WBC, this RAI is more confident to provide more reliable and realistic suggestions regarding mitigation of disruptions to the planners and managers of urban transportation.

In the future, this research could be further developed in a variety of ways. Firstly, due to the limitation of data sources, only six URNs are utilized as numerical examples. The robustness of many more URNs with different characteristics can be calculated with our RAI. Following this, other aspects of the immunity of urban transportation can be explored and quantified, such as the local disruptions which may cause node/link capacity reductions. Furthermore, although this study provides an effective tool for measuring the robustness and conducting benchmark analysis of multi-scale URNs, the reasons why some URNs show better robustness than others based on RAI are not deeply explored, and many factors are probably involved, such as travel demand, flow patterns and topology of URNs.

\section{REFERENCES}

[1] Y. Deng et al., "Deep direct reinforcement learning for financial signal representation and trading". IEEE transactions on neural networks and learning systems, vol.28, no.3, pp. 653-664, 2017.

[2] W.L. Shang, "Robustness and Resilience Analysis of Urban Road Networks". PhD Thesis of Imperial College London, 2017.

[3] W.L. Shang et al., "Statistical Characteristics and Community analysis of Urban Road Networks". Complexity. 2000.

[4] A. Nagurney, "Building Resilience into Fragile Transportation Networks in an Era of Increasing Disasters". Presented at Transportation Research Board 90th Annual Meeting, Washington D.C., USA, 2011, Available at https://supernet.isenberg.umass.edu/visuals/TRB_Panel_N agurney_Talk.pdf.

[5] W.L. Shang, Y. Chen, and W. Ochieng, "Resilience Analysis of Transport Networks by Combining Variable Message Signs With Agent-Based Day-to-Day Dynamic Learning”. IEEE Access. vol. 8, pp. 104458-104468, 2020.

[6] H. Song, R. Danda, J. Sabina, and B. Christian., "CyberPhysical Systems: Foundations, Principles and Applications". Boston, MA: Academic Press, 2016. ISBN: 978-0-12-803801-7: p. 1-514.

[7] W.L. Shang et al., "Resilience Analysis of Urban Road Networks Based on Adaptive Signal Controls: Day-to-Day 
Traffic Dynamics with Deep Reinforcement Learning”. Complexity, vol. 8841317, 2020.

[8] H. Bi et al., "GIS aided sustainable management for urban road transportation systems with a unifying queuing and neural network model". Applied Energy, 2021. 2021.

[9] J.R. Amdal. and S.L. Swigart, "RESILIENT TRANSPORTATION SYSTEMS IN A POSTDISASTER ENVIRONMEN". A Case Study of Opportunities Realized and Missed in the Greater New Orleans Region. Gulf Coast Research Center for Evacuation and Transportation Resiliency, $\quad$ http://www.evaccenter.lsu.edu/1001.pdf Accessed 12/18/2018. , 2010.

[10] IEEE, "IEEE standard Computer Dictionary : A Complation of IEEE Standard Computer Glossaries". Institute of Electrical and Electronical Engineers, 1990.

[11] B. Immers et al., "Reliability and robustness of transportation networks: problem survey and examples". Proceedings of the NECTAR cluster meeting on reliability of networks, 2004. Amsterdam, the Netherlands. pp. 19-20, 2004.

[12] S. Boccaletti et al., "Complex Networks: Structure and Dynamics”. Physics Reports, vol.424, pp. 175-308, 2006.

[13] M. Schillo et al., "Towards a definition of robustness for market-style open multi-agent systems". Proceedings of the fifth international conference on Autonomous agents. New York, pp. 75-76, 2011.

[14] P.J.G. Ribeiro and L. Goncalves, "Urban resilience: A conceptual framework". Sustainable Cities and Society, vol. $50,2019$.

[15] Y. GAO, F. Zhuo, S. Zhang, "Managing supply chain resilience in the era of VUCA". Frontiers of Engineering Management, vol.8, no.3, pp. 465-470, 2021.

[16] W.L. Shang et al., "Robustness analysis of urban road networks from topological and operational persepectives". Mathematical Problems in Engineering, vol 2020, 2020.

[17] S. Dunn, S. Wilkinson, and A. Ford, "Spatial structure and evolution of infrastructure networks". Sustainable Cities and Society, vol. 27, p. 23-31, 2016.

[18] P. Holme et al., "Attack vulnerability of complex networks". Physical Review E, vol. 65, no.5, 2002.

[19] P. Crucitti et al., "Efficiency of scale-free networks: error and attack tolerance". Physica A: Statistical Mechanics and its Applications, vol. 320, pp. 622-642, 2003.

[20] P. Crucitti et al., "Error and attack tolerance of complex networks". Physica A: Statistical Mechanics and its Applications, vol. 340, pp. 388-394, 2004.

[21] R. Ding, “Complex network theory-based urban land-use and transport interaction studies". Complexity, vol.2019, 2019.

[22] R. Albert, H. Jeong and A.L. Barabasi, "Error and attack tolerance of complex networks". Nature, vol. 406, pp. 378382, 2000.
[23] W. Shang et al., "Robustness and Topology Analysis of European Air Traffic Network Using Complex Network Theory". The 94th Transportation Research Board Annual Meeting, Washington D.C., 11-15 January 2015, 2015.

[24] D.S. Callaway et al., "Network robustness and fragility: Percolation on random graphs". Phys. Rev. Lett., vol. 85, pp. 5468-5471, 2000.

[25] P. Crucitti, V. Latora and M. Marchiori, "Model for cascading failures in complex networks". Physical Review $E$, vol.69, no.4, 2004.

[26] H. Sakakibara, Y. Kajitani and N. Okada, "Road Network Robustness for Avoiding Functional Isolation in Disasters". Journal of Transportation Engineering, vol.673, no.130, pp. 560-567, 2004.

[27] X. Sun, S. Wandelt and F. Linke, "Topological Properties of the Air Navigation Route System using Complex Network Theory. Proceedings in the 6th International Conference on Research in Air Transportation (ICRAT2014), Istanbu Technical University, Turkey, 2014.

[28] X. Wang et al., "Multi-criteria robustness analysis of metro networks". Physica A: Statistical Mechanics and its Applications, vol474, pp. 19-31, 2017.

[29] K.L. Clark et al., "Resilience of the U.S. National Airspace System Airport Network". IEEE TRANSACTIONS ON INTELLIGENT TRANSPORTATION SYSTEMS, vol.19, no.20, pp. 3785-3794, 2018.

[30] D.M. Zhang et al., "Resiliency assessment of urban rail transit networks: Shanghai metro as an example". Safety Science, vol.106, pp. 230-243, 0218.

[31] X. Yang., et al., "Energy oriented driving behavior analysis and personalized prediction of vehicle states with joint time series modelling". Applied Energy, 2020. 261: p. 114471

[32] D.M. Scott et al., "Network robustness index: a new method for identifying critical links and evaluating the perfomance of transportation networks". Journal of Transport Geography, vol.14, no.3, pp. 215-227, 2005.

[33] J.L. Sullivan et al., "Identifying critical road segments and measuring system-wide robustness in transportation networks with isolating links: A link-based capacityreduction approach". Transportation Research Part A: Policy and Practice, vol.44, pp. 323-336, 2010.

[34] A. Nagurney and Q. Qiang, "A network efficiency measure for congested networks". Europhysics Letters (EPL), vol.79, pp. 38005, 2007.

[35] A. Nagurney and Q. Qiang, "Robustness of transportation networks subject to degradable links". Europhysics Letters (EPL), vol. 80, pp. 68001, 2007.

[36] A. Nagurney and Q. Qiang, "A relative total cost index for the evaluation of transportation network robustness in the presence of degradable links and alternative travel behavior". International Trabsactions in Operation Research, vol.16: pp. 49-67, 2009. 
[37] C. Zhao, B. Fu and T. Wang, "Braess paradox and robustness of traffic networks under stochastic user equilibrium". Transportation Research Part E: Logistics and Transportation Review, vol. 61, pp. 135-141, 2014.

[38] E. Ferguson, "Transit ridership, incident effects and public policy". Transportation Research Part A: Policy and Practice, vol.26, no.5, pp. 393-407, 1992.

[39] S.C. Lo and R.W. Hall, "Effects of the Los Angeles transit strike on highway congestion". Transportation Research Part A: Policy and Practice, vpl.40, no.10, pp. 903-917, 2006.

[40] N.J.A. Van Exel and P. Rietveld, "Public transport strikes and traveller behaviour". Transport Policy, vol.8, no.4, pp. 237-246, 2001.

[41] J. Hunt, A. Brownlee and K. Stefan, "Responses to Centre Street Bridge Closure: Where the "Disappearing" Travelers Went". Transportation research record, vol.1807, pp. 5158, 2002.

[42] R.G. Clegg, "Empirical studies on road traffic response to capacity reduction". Transportation and Traffic Theory: 17th International Symposium on Transportation and Traffic Theory (ISTTT17), 2007.

[43] S. Zhu et al., "The traffic and behavioral effects of the I35W Mississippi River bridge collapse”. Transportation Research Part A: Policy and Practice, vol.44, no.10, pp. 771-784, 2010.

[44] D. Dimitriou et al., "Public Transportation during the Athens 2004 Olympics: From Planning to Performance". The 85th Transportation Research Board Annual Meeting, At Washington D.C., U.S.A., 2006.

[45] D.A. Hensher and A.M. Brewer, "Going for gold at the Sydney Olympics: How did transport perform?". Transport Reviews, vol.22, no.4, pp. 381-399, 2002.

[46] G. Giuliano and J. Golob, "Impacts of the Northridge Earthquake on Transit and Highway Use". Journal of Transportation and Statistics, vol.1, no.2, pp. 1-20, 1998.

[47] L. Wesemann et al., "Cost-of -delay studies for freeway closures caused by northbridge earthquake". Transportation Research Record, vol.1559, pp. 67-75, 1996.

[48] Q. Zhou et al., "Global Optimization of the HydraulicElectromagnetic Energy-Harvesting Shock Absorber for Road Vehicles with Human-Knowledge-Integrated Particle Swarm Optimization Scheme". IEEE/ASME Transactions on Mechatronics, vol. 26, no.3, pp. 1225-1235,2021.

[49] S. Zhu and D. Levinson, "Disruptions to Transportation Networks: A Review”. D.M. Levinson et al. (eds.), Network Reliability in Practice, Transportation Research, Economics and Policy, 2012, http://link.springer.com/chapter/10.1007\%2F978-1-46140947-2_2 Accessed 01/07/2021.

[50] W. Li and H. Song, ART: An Attack-Resistant Trust Management Scheme for Securing Vehicular Ad Hoc Networks. IEEE Transactions on Intelligent Transportation Systems, 2016. 17(4): p. 960-969.
[51] H. Song, S. Ravi, T. Sookoor, J. Sabina., "Smart Cities: Foundations, Principles and Applications". Hoboken, NJ: Wiley, 2017. ISBN: 978-1-119-22639-0: p. 1-906.

[52] P. Cui and D.Z. Li, "A SNA-based methodology for measuring the community resilience from the perspective of social capitals: Take Nanjing, China as an example". Sustainable Cities and Society, vol.53, 2020.

[53] G. Dartmann, A. Schmeink, V. Lücken, H. Song, M. Ziefle, G. Prestiflippoet, "Smart Transportation: AI Enabled Mobility and Autonomous Driving”. CRC Press, 2021. ISBN 978-0367352967.

[54] Y. Sheffi, "Urban Transporation Networks: equilibrium analysis with mathmatical programming methods". Englewood Cliffs, New Jersey: Prentice Hall, 1985.

[55] T.L. Friesz, "Dynamic Optimization and Differential Games”. International Series in Operations Research \& Management Science, Springer US, pp. 135, 2010.

[56] Bureau of Public Roads, "Traffic assignment manual. U.S. Department of Commerce", Urban Planning Division, Washington, DC., 1964.

[57] J.G. Wardrop, "Some theoretical aspects of road traffic research". Proceedings of the institution of civil engineers,Part 2, pp. 325-378, 1952.

[58] M. Beckmann, C. Mcguire and C. Winsten, "Studies in The Economics of Transportation". New Haven: Yale University Press. (see also:http:/cowles.yale.edu/sites/default/files/files/pub/misc /specpub-beckmann-mcguire-winsten.pdf), 1956.

[59] A. Nagurney, "Network economics: A variational Inequality Approach (2nd edn)". Kluwer Academic Publishers,Dordrecht, the Netherlands, 1999.

[60] W.L. Shang, K., Han, W. Ochieng, P. Angeloudis, “An Agent-Based Day-to-Day Traffic Evolution Model with Information Percolation." Transportmetrica A: Transport Science, 2017.

[61] W.L. Shang, et al., "Impacts of user behaviors and environmental benefits of bike sharing: a big data analysis". Applied Energy, vol.285, no.116429, 2021.

[62] M. Barthélemy, "Spatial networks". Physics Reports, vol.499: pp. 1-101, 2011.

[63] K.E. Lever and K. Kifayat, "Identifying and mitigating security risks for secure and robust NGI networks". Sustainable Cities and Society, vol.59, 2020.

[64] C. Giudicianni et al., "Topological Taxonomy of Water Distribution Networks”. vol.10, no.4, pp. 444,2018.

[65] T. Roughgarden, "Selfish routing and the price of anarchy. MIT Press", 2005.

[66] C. Spearman, "The proof and measurement of association between two things. American Journal of Psychology", vol.1, pp. 72-101,1904. 


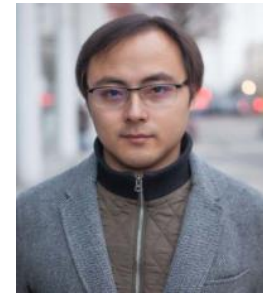

Wen-Long Shang (Member, IEEE) received the $\mathrm{Ph} . \mathrm{D}$. degree from the Centre for Transport Studies, Department of Civil and Environmental Engineering, Imperial College London. He is currently a Lecturer in the College of Metropolitan Transportation, Beijing University of Technology. He has already published nearly 30 articles in peer-reviewed journals and conferences, obtained 2 software copyrights and finished one chapters of a book. He serves as guest editors and members of editorial board for several international academic journals. He is the reviewer of nearly 30 journals and conferences. His research interests include but not limit to: green transportation, traffic big data, intelligent transport systems, emergency management, safety science and smart city.

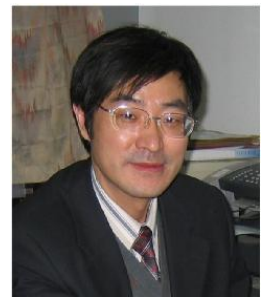

Ziyou Gao received the Ph.D. degree in operations research and control theory from the Institute of Applied Mathematics, Academia, Sinica, Beijing, China, in 1994. $\mathrm{He}$ is a Professor with the School of Traffic and Transportation, Beijing Jiaotong University. His research interests include the modeling of transportation network systems, network design problems, traffic flow theory, and dynamic traffic assignment.

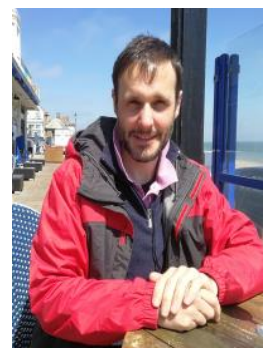

Nicolò Daina is a Lecturer and Chancellor's Fellow in Transport Policy at the University of Strathclyde, Glasgow. He received his $\mathrm{PhD}$ in Transport from Imperial College London. Before joining the University of Strathclyde, he held postdoctoral research associate and research fellow positions at Imperial College London. His research focuses on users' behavior modelling of integrated transport and energy systems. He is a member of the Transportation Research Board of the US National Academies Standing Committee on Alternative Transportation Fuels and Technologies and of the UC Davies's International EV Policy Council.

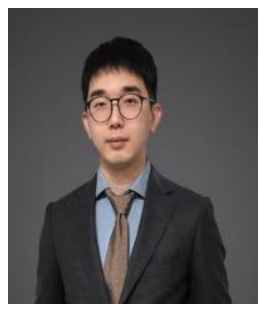

Haoran Zhang a researcher in the Center for Spatial Information Science at the University of Tokyo, and also works as a researcher at the Future Energy Center at Mälardalen University in Sweden, and Senior Scientist at Locationmind Inc. in Japan. His research includes smart supply chain technologies, GPS data in shared transportation, urban sustainable performance, GIS technologies in renewable energy systems, and smart cities. He is author of hundreds of journal articles and Editorial Board Member of several international academic journals. He has Ph.D.'s in both Engineering and Sociocultural Environment and was awarded Excellent Young Researcher by Japan's Ministry of Education, Culture, Sports, Science and Technology.

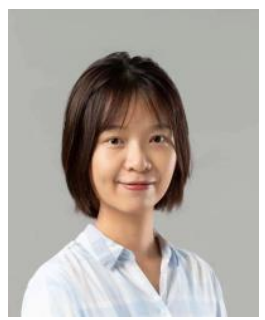

Ying Long is an assistant professor of the University of Tokyo, Japan and she has published more than 40 academic papers in high- level SCI/SSCI journals, including One earth, Applied Energy, Scientific, etc. At the same time, Dr. Long also serves as the guest editor of Frontiers in Sustainable Cities, Frontiers in Energy Research, as well as the reviewer of more than 20 high- impact academic journals. During 2013-2019, she was awarded twice by the Ministry of Education, Culture, Sports, Science and Technology of Japan as MEXT scholarship. In 2018, she was awarded as National Excellent Self-Funded Students Scholarship. In addition, Dr. Long has a multidisciplinary scientific research background and showing interests in the field of environmental economics and sustainable development.

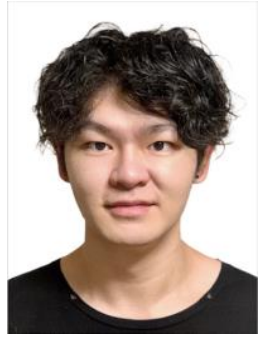

Zhiling Guo received the B.E. degree from the Chongqing University, in 2014, and the M.E. and Ph.D. degrees from the University of Tokyo, Tokyo, Japan, in 2017 and 2020, respectively. He is currently working as a data scientist in LocationMind Inc., Japan. At the same time, he received Grant-in-Aid for Young Scientists from Japan Society for the Promotion of Science (JSPS) and working as a guest researcher in the Center for Spatial Information Science (CSIS), the University of Tokyo. His current research interests include computer vision, machine learning, and geographic information science.

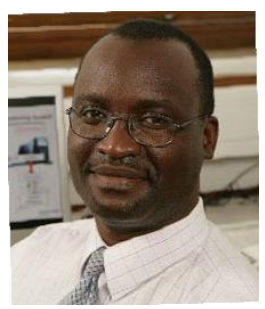

Washington Y. Ochieng is currently the Head of the Centre for Transport Studies and the Chair of positioning and navigation systems with the Department of Civil and Environmental Engineering, Imperial College London. He is also the Director of the Imperial College Engineering Geomatics Group (ICEGG). After gaining a Ph.D. in space geodesy at the University of Nottingham, he worked there as a Research Associate before moving to Racal Electronics (Thales) as the Principal Engineer (Navigation specialist), where he participated in various international industrial consortia developing satellite navigation systems and products. He moved to Imperial College London in 1997. His research interests are in the design of positioning and navigation systems for land, sea and air applications, air traffic management (ATM), and intelligent transport systems (ITS). He was the elected fellow of the Royal Academy of Engineering (FREng) in recognition of his exceptional contribution to engineering, in 2013. 